\title{
Platinum(Iv) Carboxylate Prodrug Complexes as Versatile Platforms for Targeted Chemotherapy
}

\author{
Jun Xiang Onga, Siew Qi Yapa, Daniel Yuan Qiang Wonga ${ }^{a}$, Chee Fei China, and Wee Han Ang ${ }^{\star a b}$
}

\begin{abstract}
Kinetically-inert Pt(Iv) carboxylate complexes have emerged in recent years as candidates for the development of next-generation platinum anticancer drugs. Being native prodrugs of clinically-important $\mathrm{Pt}(\mathrm{I})$ chemotherapeutic agents, the Pt(IV) scaffold can be exploited to incorporate additional functionalities while keeping the Pt(II) pharmacophore intact. This mini-review examines recent work performed to illuminate the mechanism of $\mathrm{Pt}$ (Iv) prodrug activation and their use as versatile platforms for targeted chemotherapy.
\end{abstract}

Keywords: Anticancer · Cisplatin · Oxaliplatin · Platinum(IV) prodrugs · Targeted chemotherapy

\section{Introduction}

Anticancer Pt(II) drugs such as cisplatin and oxaliplatin constitute an important class of chemotherapeutic agents that are being used clinically against many types of cancers (Fig. 1). ${ }^{[1]}$ Yet these drugs are associated with side-effects and toxicity, arising from premature aquation and high chemical reactivity, which severely limit their clinical effectiveness. ${ }^{[2]} \mathrm{Pt}$ (IV) carboxylate complexes, based on anticancer Pt(II) drug motifs, can mitigate some of these limitations being kinetically and substitutionally inert and hence, less susceptible to aquation and reactivity with nucleophiles. ${ }^{[3]}$ Within an intracellular environment, the Pt(IV) scaffold can undergo chemical reduction to its reactive $\mathrm{Pt}(\mathrm{II})$ congener, with cleavage of its axial ligands, thereby uncaging its cytotoxic potential. Currently, satraplatin represents the most successful Pt(IV) prodrug design, and exhibits an anti-proliferative profile that is distinct from cisplatin (Fig. 1). ${ }^{[4]}$ It is being evaluated in several clinical trials against hormone-refractory prostate cancer as well as advanced solid tumors. ${ }^{[5]}$ This mini-

${ }^{\star}$ Correspondence: Dr. W. H. Ang ${ }^{\text {ab }}$

E-mail: chmawh@nus.edu.sg

aDepartment of Chemistry

National University of Singapore

3 Science Drive 3, Singapore 117543

bNUS Graduate School of Integrative Sciences and

Engineering

Centre for Life Sciences (CeLS)

28 Medical Drive, Singapore 117456 review examines some of the recent work that was done in the context of understanding the mechanism of the Pt(IV) prodrug activation and their exploitation as versatile platforms for targeted chemotherapy.

\section{Activation by Reduction: Evidence for a Prodrug Mechanism}

The widely-held view is that $\mathrm{Pt}(\mathrm{IV})$ carboxylate complexes are themselves not pharmacologically active but require chemical reduction to their $\mathrm{Pt}(\mathrm{II})$ congeners which are the active cytotoxic species. ${ }^{6]}$ This is in part based on the observation that $\mathrm{Pt}(\mathrm{IV})$ complexes are inert towards DNA, the presumed biological target, whereas $\mathrm{Pt}(\mathrm{II})$ complexes readily bind them. For example, we have showed using reverse phase liquid chromatography (RPLC) that Pt(IV) carboxylate prodrug complexes based on the cisplatin template do not bind dGMP (as a model nucleo- philic nucleobase). Yet in the presence of a reductant, such as ascorbic acid, these Pt(IV) complexes formed Pt-dGMP adducts akin to those formed when cisplatin reacted directly dGMP. ${ }^{[7]}$ Further evidence from immunofluorescence experiments, showed that $\mathrm{Pt}(\mathrm{IV})$ prodrug based on the cisplatin template formed similar nuclear Pt-DNA adducts when exposed to cancer cells in vitro. ${ }^{[8]}$ It therefore follows that in order to function as anticancer prodrugs, Pt(IV) carboxylate complexes undergo trans-cleavage of both axial ligands to liberate Pt(II) species. However, it has also been suggested that reduction may actually proceed via multiple pathways leading to the loss of any combination of the axial carboxylate and equatorial chloride ligands to yield a variety of possible reduction products. ${ }^{[9]}$

To investigate the nature of this reduction process, we employed a combination of hydrophilic interaction chromatography (HILIC) and RPLC to probe the reduction

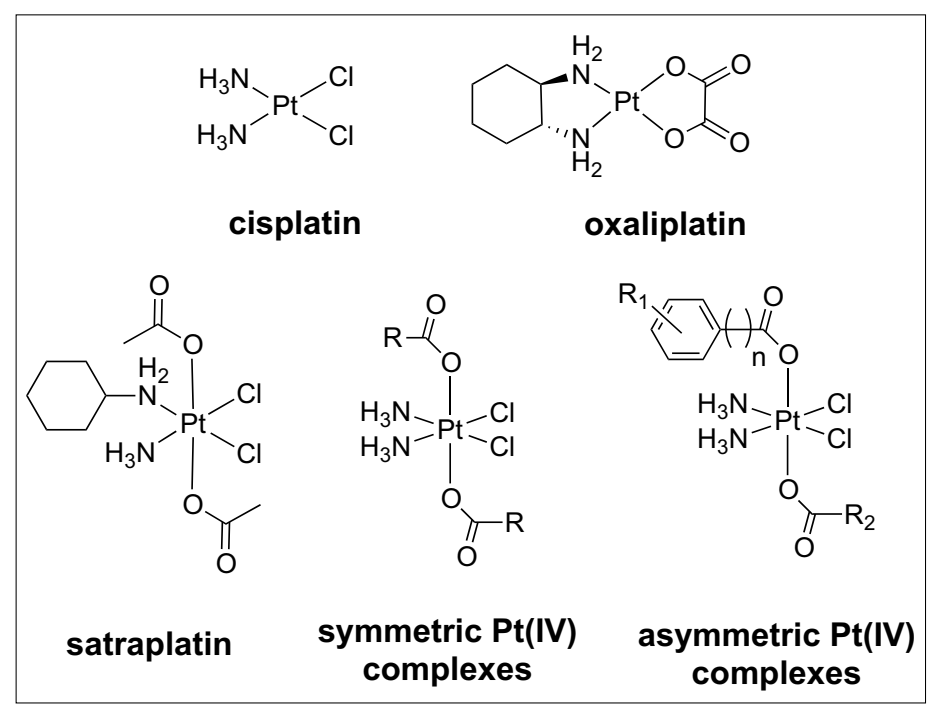

Fig. 1. Molecular structures of $\mathrm{Pt}(\mathrm{II})$ drugs and Pt(IV) prodrug complexes. 
of Pt(IV) prodrug complexes based on the cisplatin template using ascorbic acid as a model of an outer-sphere reductant. ${ }^{[10]}$ Because HILIC and RPLC possessed orthogonal retention for analytes, cisplatin (the primary reduction product of interest) would be well-resolved on HILIC even though it could not be resolved by RPLC. Other reduction products could also be systematically identified. These complementary chromatographic techniques showed that such Pt(IV) complexes were predominantly reduced to cisplatin $(>89 \%)$ and satraplatin to cis-(ammine)(hexylamine) dichloroplatinum(II) (>95\%), thus qualifying them as true prodrugs of the respective Pt(II) pharmacophore. Reaction kinetics showed that the subtle substitution of an axial carboxylate ligand with a hydroxide group, i.e. a $\mathrm{Pt}(\mathrm{IV})$ monocarboxylate complex, drastically enhanced reduction rates leading to highly reducible Pt(IV) prodrug complexes.

We are also interested to examine how these $\mathrm{Pt}$ (IV) prodrugs are being taken up and activated within living cells in vitro. The main challenge is that existing elemental imaging techniques centred on Pt detection e.g. inductively-coupled plasma mass spectrometry (ICP-MS), secondary ion mass spectrometry (SIMS) imaging, cannot distinguish between $\mathrm{Pt}(\mathrm{IV})$ and $\mathrm{Pt}(\mathrm{II}) .{ }^{[11]} \mathrm{X}$-ray photoelectron spectroscopy can directly determine $\mathrm{Pt}(\mathrm{II})$ and $\mathrm{Pt}(\mathrm{IV})$ levels in cryogenically-preserved cell lysates but it cannot be applied on live cells and is currently incompatible with cellular imaging. ${ }^{[12]}$ To address these challenges, we developed a fluorogenic probe that would be specifically activated by Pt(II) drug moieties under physiological conditions and therefore compatible with high resolution fluorescence microscopy imaging. ${ }^{[13]}$ The probe was based on a non-fluorescent rhodamine spirolactam construct linked to a dithiocarbamate motif that reacted readily with $\mathrm{Pt}(\mathrm{II})$ complexes; binding of $\mathrm{Pt}(\mathrm{II})$ at dithiocarbamate and the proceeding reaction with the spirolactam led to spiro-ring opening and fluorescence turn-on (Fig. $2 \mathrm{a})$. These fluorogenic probes are inert towards the parental Pt(IV) complex (Fig. 2b) as well as Pt(II) species bearing chelating amine ligands e.g. oxaliplatin. We showed for the first time that $\mathrm{Pt}(\mathrm{IV})$ prodrug complexes, including satraplatin, were being endogenously reduced intracellularly within the cytoplasm following cell entry and uptake.

\section{Asymmetric Pt(Iv) Carboxylate Complexes with Finely Tuned Properties}

From a design perspective, the $\mathrm{Pt}(\mathrm{IV})$ scaffold provides an excellent platform to attach functionalities while retaining the Pt(II) pharmacophore. Enzyme inhibitors and moieties that can potentiate cisplatin activity have been added as axial ligands to the $\mathrm{Pt}$ (IV) scaffolds to induce synergistic effects. ${ }^{[14]}$ Structure-activity relationship studies have shown that the redox potential, rate of reduction, lipophilicity, and cytotoxicity of platinum(IV) prodrugs could be altered by varying the axial ligands. ${ }^{[15]}$ However, classical approaches to prepare symmetrical Pt(IV) complexes with the same carboxylate ligands in both axial positions are too limited to fully exploit the vast potential of this prodrug strategy. We therefore devised a practical way to access asymmetrical Pt(IV) complexes with different axial ligands (Fig. 1). The broad strategy was to employ mildly electrophilic carboxylation reagents under highly dilute conditions in order to achieve monocarboxylation of the Pt(IV)-dihydroxide precursor. The remaining axial position was then sequentially carboxylated to arrive at the desired asymmetrical Pt(IV) complex. ${ }^{[16]}$ As a proof-of-method, we prepared Pt(IV) carboxylate complexes using ligands with contrasting attributes, e.g. one lipophilic and one hydrophilic, that would not be feasible under classical carboxylation conditions. ${ }^{[7]}$ Since Pt(IV) prodrugs would assume the properties of their coordinating ligands, the different axial ligands served as handles to fine-tune the pharmacological properties of the overall prodrug complex. Hence by altering their aqueous solubilities and lipophilicities, we developed a series of asymmetric Pt(IV) carboxylate prodrug complexes with finely-tuned pharmacological properties in the context of enhancing their antiproliferative efficacies in vitro.

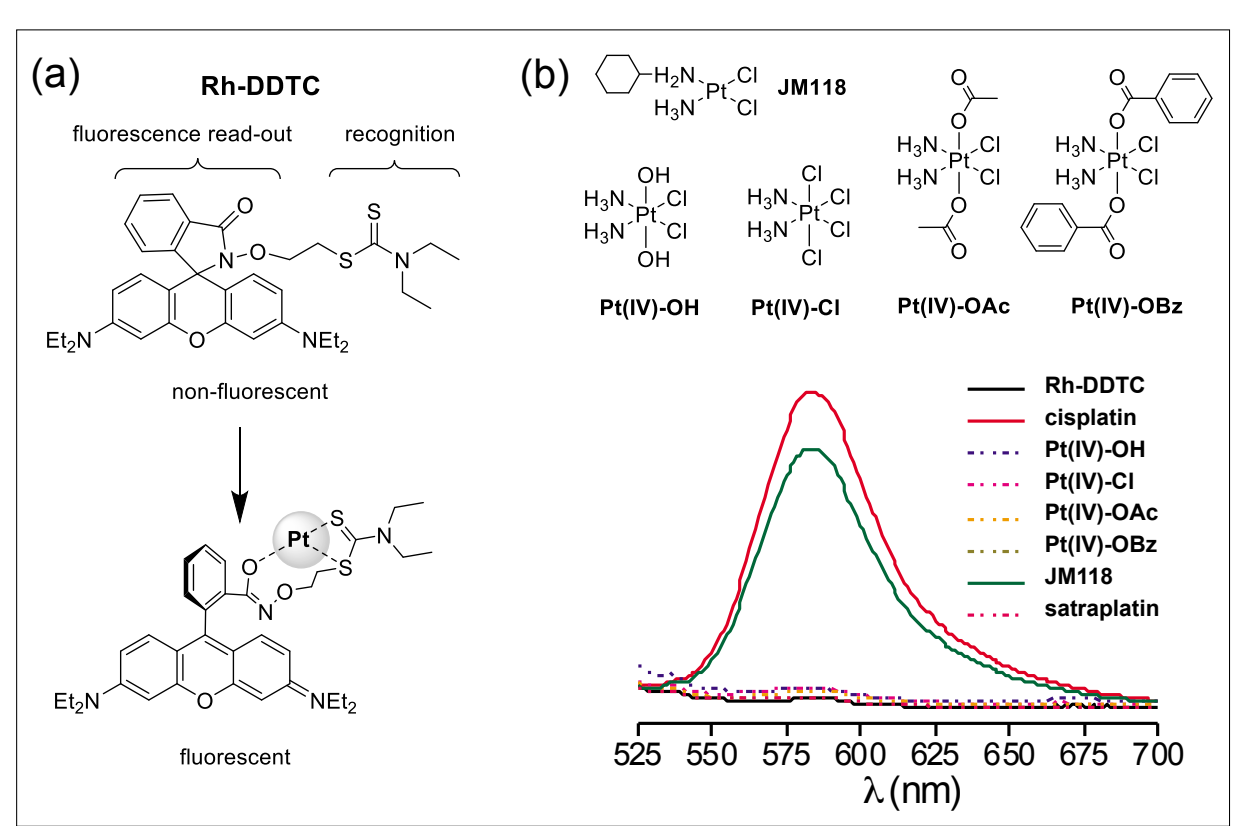

Fig. 2. (a) Mechanism of activation of Pt(II)-selective fluorogenic probe Rh-DDTC; (b) fluorescence

profile of Rh-DDTC exposed to different Pt compounds.

\section{Facile Preparation of Pt(Iv)- Peptide Complexes through Chemoselective Oxime Ligation}

Preparing Pt(IV) prodrug complexes via direct axial carboxylation is not always possible due to the lack of activated electrophilic carboxylation reagents for the desired functional groups.[17] An alternative strategy is to conjugate the functional groups to the $\mathrm{Pt}(\mathrm{IV})$ scaffold through a pendant carboxylic moiety across a succinate linker. Conjugation can be readily carried out using amide or ester coupling chemistry, but yields are generally low and product purification is complicated by the presence of accompanying coupling reagents and by-products. ${ }^{[18]}$ To address this limitation, we sought to develop a facile conjugation strategy that would be applicable to $\mathrm{Pt}(\mathrm{IV})$ carboxylate chemistry (non-reducing conditions) and compatible with biologically relevant molecules such as peptides (mild reaction conditions) with the goal of harnessing them to enhance the selectivity of $\mathrm{Pt}$ prodrugs. To that end, we showed that oxime ligation chemistry using a benzaldehyde-functionalized $\mathrm{Pt}$ (IV) precursor and a hydroxylamine-functionalized peptide in slightly acidic conditions could generate Pt(IV)-peptide conjugates quantitatively under ambient conditions (Fig. 3). ${ }^{[19]}$ Because the hydroxylamine functional groups could be readily incorporated as part of peptide solid-phase synthesis, either at the $N$-terminus or incorporated into an amino acid residue, such functionalised peptides could be made commercially available. Reaction yields were high and because the reaction protocol involved only stoichiometric amounts of hydroxylamine-functionalized peptide 
and $\mathrm{Pt}(\mathrm{IV})$ precursor in buffer, product purification through RPLC was facile.

We applied this generic strategy in the development of Pt(IV)-peptide conjugates under two different contexts. Firstly, we prepared $\mathrm{Pt}(\mathrm{IV})$ conjugates containing formyl peptide receptor $1 / 2$ (FPR1/2)targeted peptides. Pt-based anticancer drugs were shown to be potent immunomodulators of both the innate and the adaptive immune system but there was little in the way of exploiting their immune-activating properties in rational drug design. FPR1/2 receptors are highly expressed in immune cells as well as some metastatic cancers. We therefore tethered a dual-purpose peptide sequence, which behaved as both a FPR1/2-targeting moiety and an immune adjuvant, to the Pt(IV) prodrug scaffold containing the cisplatin template. We demonstrated that these Pt(IV) conjugates not only induced targeted cytotoxicity in FP1/2-expressing cancer cells but they were also capable of potentiating the cellmediated cytotoxicity of peripheral blood mononuclear cells (PBMCs) against cancer cells. In keeping with the immune-activation mechanism, elevated extracellular secretion of pro-inflammatory cytokines TNF- $\alpha$ and IFN- $\gamma$ in treated PBMCs were also observed. Thus, these Pt(IV)-peptide conjugates functioned as rationally-designed chemo-immunotherapeutic agents with multiple modes of action. ${ }^{[10]}$

More recently, we extended this strategy to prepare $\mathrm{Pt}(\mathrm{IV})$-peptide conjugates, based on the cisplatin and oxaliplatin templates, targeting human epidermal growth factor receptor 2 (HER2/neu), a clinically-validated and well-studied biological target. ${ }^{[20]}$ These Pt(IV)-peptide conjugates target highly HER2-expressing cells, in the presence of basal HER2-expressing cells, and are strongly taken up intracellularly. Intriguingly, they induced necrotic cell death instead of apoptotic cell death as a result of the massive Pt influx. We further showed that this strategy of targeted necrosis could be harnessed to overcome p53-dysfunctional cells that were resistant to apoptosis.

\section{Hydrophobic Entrapment/Reversal as Strategy for Controlled-release Pt(Iv)-Nanoconstructs}

Rigid nanomaterials including nanosheets, nanotubes and nanohorns have been widely explored as potential drug delivery platforms. Their structural rigidity enables them to be precisely shaped, which can then be exploited for specific biological interactions. For example, carbon nanotubes/ nanohorns have high aspect ratios which allow them to breach and penetrate the outer cellular membrane without inducing

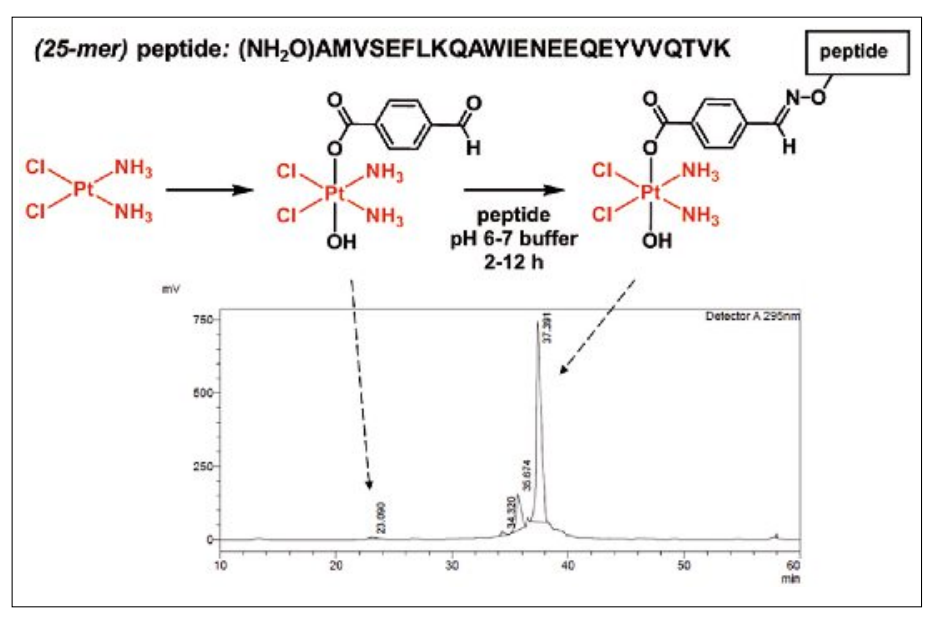

Fig. 3. Representative reactivity of $\mathrm{Pt}(\mathrm{IV})$ benzaldehyde with hydroxylamine-functionalised peptide; analytical RPLC chromatogram of crude reaction mixture at 1:1 stoichiometric ratio.

cytotoxicity. ${ }^{[21]}$ In addition, their hollowed hydrophobic interior cavity can be used to entrap drug entities within the tube walls providing a protective barrier. ${ }^{[22]}$ However, the main limitation in applying them as drug delivery platforms is that entrapped hydrophobic drugs will be highly stable within such an environment and not amenable to release. Hydrophilic drugs, on the other hand, would be unstable within the hydrophobic cavity and readily displaced by competing solvent molecules.

To solve this problem, we engineered the controlled release mechanism into the $\mathrm{Pt}(\mathrm{IV})$ prodrug scaffold based on the concept of hydrophobic reversal on reduction. In its inactive prodrug form, the Pt(IV) complex would be highly hydrophobic by design $(\log P=+2.19)$ and readily entrapped within multiwalled carbon nanotubes (CNTs).[23] After cell penetration, the $\mathrm{Pt}(\mathrm{IV})$ prodrug could be reduced to hydrophilic cisplatin $(\log P=-2.28)$ and readily extruded from the hydrophobic cavity. Because chemical reduction would occur after cell entry, controlled release by intracellular reduction and subsequent hydrophobicity reversal was achieved (Fig. 4). We further showed that Pt release was dependent on the solvent accessibility at the nanotube entrances since reduction was mediated by intracellular reductants; hence, a crowded entrance resulted in slower Pt release. ${ }^{[24]}$ Furthermore, the rigid CNT platform could be readily functionalized with targeting groups enabling selective uptake.[25] More recently, we showed that the Pt(IV) scaffold could be modified to carry another drug entity such as doxorubicin and that the combined prodrug could be stably entrapped within multiwalled CNTs.[26] This provided a proofof-concept of a strategy to deliver ratiometric drug combinations using cRGDfKfunctionalised multiwalled CNTs in which drug entities were synchronously activated and released within targeted cells.

\section{Conclusion}

In summary, $\mathrm{Pt}(\mathrm{IV})$ carboxylate prodrug complexes are highly versatile platforms for developing targeted chemotherapeutic strategies capable of overcoming existing limitations of classical Pt(II) drugs. Recent progress enabling specific functionalization of Pt(IV) prodrug complexes as well as advances towards understanding their cellular processing pave the way for their development into clinically-relevant agents for cancer therapy.

\section{Acknowledgements}

Financial support from the National University of Singapore and Ministry of Education MOE Tier 2 (R143-000-572-112) is gratefully acknowledged.

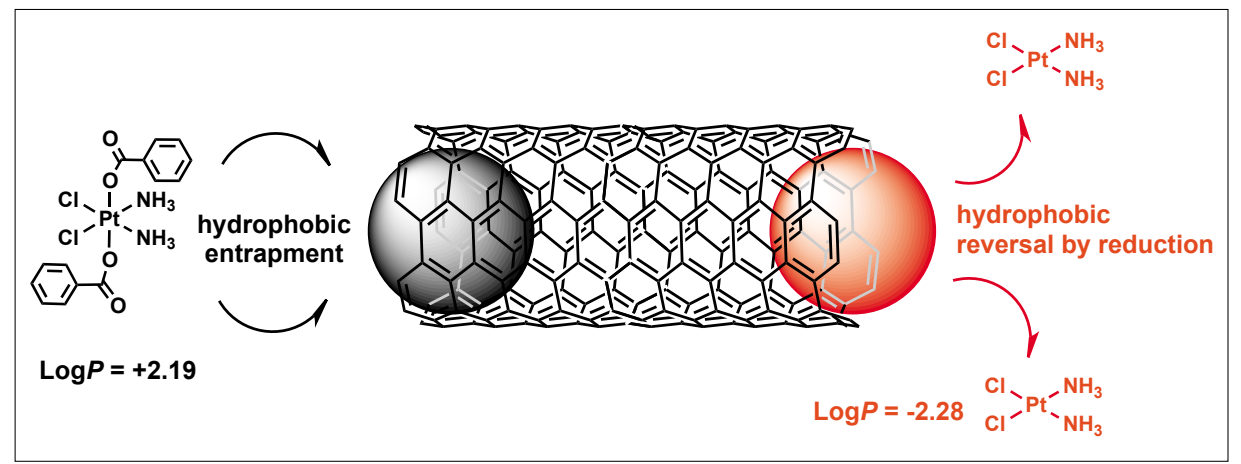

Fig. 4. Concept of hydrophobic entrapment of Pt(Iv) prodrug complexes in multiwalled CNTs entrapping; controlled release via hydrophobic reversal by intracellular chemical reduction. 
Received: January 10,2015 [11] a) R. A. Alderden, H. R. Mellor, S. Modok, M. D. Hall, S. R. Sutton, M. G. Newville, R. Callaghan, T. W. Hambley, J. Am. Chem. Soc. 2007, 129, 13400; b) A. R. Timerbaev, S. S. Aleksenko, K. Polec-Pawlak, R. Ruzik, O. Semenova, C. G. Hartinger, S. Oszwaldowski, M. Galanski, M. Jarosz, B. K. Keppler, Electrophoresis 2004, 25, 1988; c) L. E. Wedlock, M. R. Kilburn, R. Liu, J. A. Shaw, S. J. Berners-Price, N. P. Farrell, Chem. Commun. 2013, 49, 6944.

[12] M. D. Hall, G. J. Foran, M. Zhang, P. J. Beale, T. W. Hambley, J. Am. Chem. Soc. 2003, 125 , 7524.

[13] D. Montagner, S. Q. Yap, W. H. Ang, Angew. Chem. Int. Ed. 2013, 52, 11785.

[14] a) W. H. Ang, I. Khalaila, C. S. Allardyce, L. Juillerat-Jeanneret, P. J. Dyson, J. Am. Chem. Soc. 2005, 127, 1382; b) W. H. Ang, S. Pilet, R. Scopelliti, F. Bussy, L. Juillerat-Jeanneret, P. J. Dyson, J. Med. Chem. 2005, 48, 8060; c) K. R. Barnes, A. Kutikov, S. J. Lippard, Chem. Biol. 2004, 11, 557.

[15] a) S. Choi, C. Filotto, M. Bisanzo, S. Delaney, D. Lagasee, J. L. Whitworth, A. Jusko, C. Li, N. A. Wood, J. Willingham, A. Schwenker, K. Spaulding, Inorg. Chem. 1998, 37, 2500; b) J. J. Wilson, S. J. Lippard, Inorg. Chem. 2011, 50 , 3103.

[16] J. Z. Zhang, P. Bonnitcha, E. Wexselblatt, A. V. Klein, Y. Najajreh, D. Gibson, T. W. Hambley, Chem. Eur. J. 2013, 19, 1672.
[17] M. Galanski, B. K. Keppler, Inorg. Chem. 1996, 35, 1709.

[18] a) S. Mukhopadhyay, C. M. Barnes, A. Haskel, S. M. Short, K. R. Barnes, S. J Lippard, Bioconjugate Chem. 2008, 19, 39; b) M. Reithofer, M. Galanski, A. Roller, B. K. Keppler, Eur. J. Inorg. Chem. 2006, 2612.

[19] D. Y. Q. Wong, J. Y. Lau, W. H. Ang, Dalton Trans. 2012, 41, 6104.

[20] D. Y. Q. Wong, J. H. Lim, W. H. Ang, 2015 , submitted.

[21] B. S. Wong, S. L. Yoong, A. Jagusiak, T. Panczyk, H. K. Ho, W. H. Ang, G. Pastorin, Adv. Drug Deliv. Rev. 2013, 65, 1964.

[22] a) J. Li, S. Q. Yap, S. L. Yoong, T. R. Nayak, G. W. Chandra, W. H. Ang, T. Panczyk, S. Ramaprabhu, S. K. Vashist, F. S. Sheu, A. Tan, G. Pastorin, Carbon 2012, 50, 1625; b) J. Li, A. Pant, C. F. Chin, W. H. Ang, C. Menard-Moyon, T. R. Nayak, D. Gibson, S. Ramaprabhu, T. Panczyk, A. Bianco, G. Pastorin, Nanomed. Nanotechnol. Biol. Med. 2014, 10, 1465.

[23] J. Li, S. Q. Yap, C. F. Chin, Q. Tian, S. L. Yoong, G. Pastorin, W. H. Ang, Chem. Sci. 2012, 3, 2083.

[24] L. Muzi, C. Menard-Moyon, J. Russier, J. Li, C. F. A. Chin, W. H. Ang, G. Pastorin, G. Risuleo, A. Bianco, Nanoscale 2015, in press.

[25] S. L. Yoong, B. S. Wong, Q. L. Zhou, C. F. Chin, J. Li, T. Venkatesan, H. K. Ho, V. Yu, W. H. Ang, G. Pastorin, Biomaterials 2014, 35, 748 .

[26] C. F. Chin, S. Q. Yap, J. Li, G. Pastorin, W. H. Ang, Chem. Sci. 2014, 5, 2265. 